\title{
A mobility model for classical swine fever in feral pig populations
}

\author{
George Milne*, Chloe Fermanis, Paul Johnston \\ School of Computer Science and Software Engineering, The University of Western Australia, Australia
}

(Received 30 August 2007; accepted 10 July 2008)

\begin{abstract}
We present a simulation model which explicitly captures the movement of wild animals over the landscape and the effect which herd mobility has on the temporal and spatial course of an epidemic. Using the example of classical swine fever in feral pig populations in the tropical savannas, we demonstrate that seasonal factors influencing population density and movement patterns are an important factor in the transmission of the disease. Pig population density is much greater at the start of the dry season than at the start of the wet season, with an epidemic most likely to occur if initiated at the start of the dry season. Spatial heterogeneity due to scarcity of water in the dry season causes herds to congregate around water sources. This concentration of herds, and the consequential isolation of sub-populations, reduces overall disease transmission compared with a model where the population is more evenly distributed over the landscape. The presence of adult male pig herds, which travel over greater distances than family herds, is shown to increase the overall scale of an outbreak in the dry season by connecting together otherwise isolated family herds. Eradication strategies are more likely to be successful in the dry season if they target long-range adult male herds. Our simulation method is generic and is equally applicable to other diseases where the host species is mobile.
\end{abstract}

classical swine fever / disease modelling / simulation / spatial model / feral and wild animal populations

\section{INTRODUCTION}

Significant research has occurred to improve simulation models with which to estimate disease spread in domestic animal populations and to examine the effect of various interventions in eradicating disease outbreaks, in part driven by the 2001 footand-mouth disease outbreak in the United Kingdom. However, much less attention has been given to modelling the time-course of diseases in wild animal populations. Furthermore, these populations may have movement characteristics and population dynamics which vary seasonally. The aim of the research reported here has been to develop appropriate modelling techniques to capture the effects which seasonality and variable animal mobility characteristics have on the

*Corresponding author: milne@csse.uwa.edu.au growth rate and spatial extent of a highly transmissible disease. We illustrate these new techniques using classical swine fever in feral pig populations in the tropical savanna of northern Australia, a geographical region with very distinct wet and dry seasons.

Spatial simulation models have been developed in order to understand the time-course and changing spatial scale of an outbreak and the effect which targeted culling or vaccination may have [4, 11, 20-22]. These models are generally focused on domestic animals, with some models capturing explicit animal movement between farms, saleyards and other locations where disease transmission may readily occur. The significant movement of animals between different farms via markets was a key factor in the rapid, country-wide dissemination of foot-and-mouth disease in the United Kingdom in 2001 [10,21,26]. 
In some countries, diseases may be transmitted between domestic and wild animal populations, either indirectly via locations where live viruses survive in the soil, around water sources for example, or by direct contact between animals from these two sub-populations. Wild or feral animal populations may act as disease reservoirs which may continuously spill over to the domestic animal population, making control and eradication significantly more difficult than if the disease were to reside solely in the domestic sub-population [29]. For example, a foot-and-mouth outbreak in deer in California in 1924 required extensive efforts over a two year period to eradicate the disease in a single national park $[17,24]$. It is thus pertinent to develop simulation models which allow for an examination of the mechanisms underlying the potential for disease transmission between domestic and wild animals. A challenge faced in this task is to capture the movement characteristics of the wild animal subpopulation. It is this spatio-temporal mobility which contributes substantially to long-range spread of animal diseases [4,26].

Domestic animal populations may be readily modelled using accurate records of animals located on farm premises, with some countries (e.g. Denmark) also recording animal movements among farms and 'processing plants'. However, animal population, distribution and movement data on wild or feral animal populations is scarce. Recent work [7, 17,33] uses spatial models to estimate transmission of foot-and-mouth disease among feral pig and deer populations, in Queensland, Australia and Texas, USA. These researchers utilise a cellular automata technique, dividing the landscape into a discrete grid of cells [12]. Individual susceptible cells may become infected, with disease transmission crossing the landscape by a percolation process among neighbouring cells. In contrast to this approach, we model herd (rather than location) infectivity together with explicit herd mobility, as is done in recent simulation models for pandemic influenza [9, 14, 15, 23]. The ability to explicitly model herd infectivity and mobility allows us to capture variable mobility characteristics, such as found during the wet and dry seasons in the tropics where rainfall patterns differ significantly.

Feral pigs ${ }^{1}$ are used as the animal species with which we illustrate this modelling approach and classical swine fever as the disease of interest. The concepts presented are applicable to other species and other communicable diseases. Using this method we examined the effect which seasonal factors have on the spread of classical swine fever (CSF) and how such seasonal factors may then impact on subsequent eradication strategies. The lack of accurate information regarding the distribution and movement characteristics of feral animal populations caused us to examine probable characterising scenarios for feral pig populations and to develop suitable modelling techniques for each of them.

Classical swine fever is a contagious List A disease ${ }^{2}$ which may spread rapidly through both feral and domestic pig populations. If the disease enters the feral pig population disease eradication requires a difficult and costly program of surveillance and response [2]. Outbreaks of classical swine fever in domestic animal populations, such as occurred in the Netherlands in 1997-1998 [19], are controlled by movement bans and eradicated by culling; while costly to the farming industry, such eradication schemes are effective. In countries with large feral pig populations, such as Australia with an estimated 20 million, eradication of CSF will be significantly more difficult if the disease were to enter this population. Direct or close contact between susceptible and infected animals is the common route by which the CSF virus is transmitted and is the focus of in the study reported here. The virus can also be spread via aerosol transmission but this is only of epidemiological significance when animals are confined to small areas, as in domestic pig premises [19].

\footnotetext{
${ }^{1}$ Sus scrofa when not domesticated may be referred to as feral pig or wild boar.

${ }^{2}$ OIE, International Animal Health Code: Section 2.1 [on line] (2007) http://www.oie.int/eng/normes/ mcode/en_chapitre_2.1.1.htm, OIE, Paris, 2007 [consulted 09/07/2008].
} 
Feral pigs form small social groups of four to ten animals, with sow offspring groups comprising two to four sows with their litters and bachelor groups consisting solely of males. Feral pigs confine their movements to a defined home range, the size being primarily determined by the availability of food. Female pigs roam within a home range of 2 to $20 \mathrm{~km}^{2}$ while adult males have larger home ranges than sows, averaging between 8 and $50 \mathrm{~km}^{2}[5,16]$. This heterogeneous organization of feral pig herds is significant for CSF epidemiology as it affects the frequency of contact between herds.

Feral pigs do not move quickly, with their daily movement averaging around $1 \mathrm{~km}^{2}$ [28]. For example, most observed movements of feral pigs in tropical northern Australia appear to be seasonal and relate to food sources which vary between the wet and dry seasons. In Cairns, north Queensland the average rainfall from December to May is $1740 \mathrm{~mm}$ while in the dry season it is $280 \mathrm{~mm}^{3}$ with consequential food scarcity, resulting in increased herd movement compared with the wet season ${ }^{4}$. Pig density in northern Australia also varies seasonally with 2 to $3.2 \mathrm{pigs} / \mathrm{km}^{2}$ towards the end of the dry season and 9.5$12.3 \mathrm{pigs} / \mathrm{km}^{2}$ towards the end of the wet season $[5,18]$. This is due to higher mortality rates amongst sows and young pigs resulting from limited food supplies during the latter part of the dry season.

Classical approaches to disease modelling simulate the host population as a single entity and have generally not incorporated spatial aspects of the epidemic process. Due to the significance to disease spread of spatial heterogeneity and movement on the landscape, spatially explicit models and their attendant simulation software have been developed, such as those developed for footand-mouth disease $[4,10,11,21,26,32]$. These models are used to examine the effects of

\footnotetext{
${ }^{3}$ Australian Bureau of Meteorology.

${ }^{4}$ Agriculture and Resource Management Council of Australia and New Zealand, Disease strategy: classical swine fever, Australian veterinary emergency plan, 1996.
}

various containment or eradication strategies, namely culling, vaccination and movement bans. Spatial simulation models based on cellular automata have also been developed for wild [7, 17, 33] and domestic [12] animal populations.

The aim of our study has been to develop new modelling techniques which are directly applicable to wild, freely-roaming animal populations and the effects which seasonality has on population size and mobility. These techniques are illustrated using the spatial dynamics of CSF outbreaks in feral pig populations in the tropical savannas, where we determine how such outbreaks spread through time and space as affected by the substantial seasonal variation in host population dynamics. In the simulation model presented here we make movement explicit, allowing us to capture variation in mobility for different host types (in the CSF case we have adult male pig herds roaming further than family groups). Furthermore, we demonstrate how explicit mobility allows for the representation of movement dynamics which are directly influenced by the environment, such as the attraction of pig herds to water sources during the tropical dry season. To address the uncertainty of feral pig movement characteristics [13], the flexibility of this animal movement modelling approach has been used to develop alternative movement scenarios and to examine how an outbreak would progress under each of them. While our study relates to CSF and feral pigs, our method may be applied to other diseases, such as foot-and-mouth disease and to other animal populations including migratory wildlife.

\section{MATERIALS AND METHODS}

\subsection{Model description}

The model consists of: a gridded landscape of $1 \mathrm{~km}^{2}$ cells with some containing permanent water sources (see Figure 1); herds having a type (adult male or family), home-range, current location and current disease state, susceptible $(S)$, exposed $(E)$, infectious $(I)$, recovered $\left(R_{1}\right)$ or removed $\left(R_{2}\right)$ [22]; disease transmission and disease progress rules; and herd movement rules.

(page number not for citation purpose) Page 3 of 11 

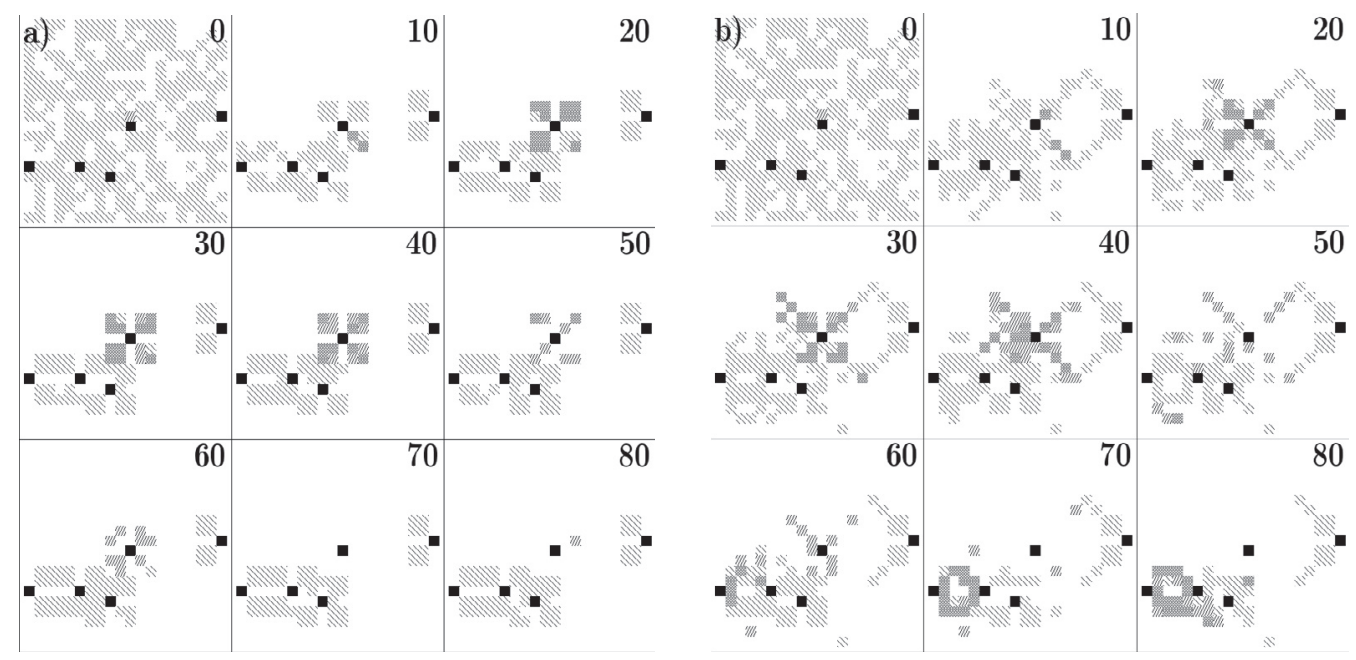

Figure 1. Map of herd locations at 10 day intervals for the water seeking movement model where (a) there are no adult male herds; (b) $30 \%$ are adult male herds and the remainder family herds. The five water sources are coloured black. The size of the model world is $20 \mathrm{~km} \times 20 \mathrm{~km}$. Non-water cells with susceptible herds present are shaded top left to bottom right $(\backslash)$, cells with infectious herds are shaded bottom left to top right (/) and cross-hatching is used where both susceptible and infectious states are present. Initially herds are randomly located and keep moving until they find a water source, which can be seen to have occurred by day 10. Once a water location is found, the home range is centred at this location and the herd may then move within the 25 (family herds) or 81 (adult male herds) cells centred on the water source.

The proportion of each herd type (adult male or family) and the home-range for each herd type are model parameters. The initial location of each herd is random with all but one herd in the susceptible disease state. An infectious index herd is placed at the centre of the modelled landscape.

Transitions between disease states occur in a probabilistic manner according to the following rules. The transition of a herd from a susceptible to an exposed state may occur when susceptible and infectious herds are simultaneously co-located in the same cell location. For each susceptible herd its probability of becoming infected is given by:

$$
P(S \rightarrow E)=T \frac{n_{I}}{n-1}
$$

where $(0<T \leq 1), n_{I}$ and $n$ are the number of infectious herds and the total number of herds (excluding removed herds) in the cell, respectively. The transmission probability $T$ is the probability that a contact between a susceptible and infectious herd results in disease transmission.

Once a herd is infected it remains in the exposed state for a fixed incubation period and then changes state to the infectious $(I)$ state. An infectious herd remains in this state for a fixed duration, at the completion of which the herd either enters the recovered $\left(R_{1}\right)$ or removed $\left(R_{2}\right)$ state, with probabilities:

$$
P\left(I \rightarrow R_{1}\right)=1-M, \quad P\left(I \rightarrow R_{2}\right)=M
$$

where $M$ is the herd mortality probability. A herd in the removed state remains in that state. Recovered herds also remain in that state to reflect the fact that immunity lasts for at least six months [2], which coincides with the duration of our simulations. Each herd moves to a neighbouring cell every day, or every second day if infectious, for a movement rate of $1 \mathrm{~km}$ per day [5].

\subsection{Water seeking behaviour}

Movement patterns governed by the presence and location of water sources are particularly significant to feral pigs during the dry season in the tropics when they are unable to satisfy their water requirements by consuming vegetation alone. 
Table I. Model parameters used in simulations. The bold values are the standard values. Other values are used either for the sensitivity analyses in Figure 3 or for the simulation results presented in Table II and Table III

\begin{tabular}{|c|c|}
\hline Parameter & Values - standard value is bold \\
\hline Herd density (i.e. average number of herds per cell) & $0.25,0.5,0.7, \mathbf{1 . 0}, 2.0$ \\
\hline Number of cells in modelled world & 400 \\
\hline Transmission probability (of effective contacts that result in infection) & $30, \mathbf{7 0}, 100 \%$ \\
\hline Exposed period & $0,4, \mathbf{8}$ days \\
\hline Infectious period & $\mathbf{1 0}, 20,30,60$ days \\
\hline Adult male herd percentage & $\mathbf{0 \%}, 15 \%, 30 \%$ \\
\hline Herd mortality probability & $10 \%$ \\
\hline Family herd range & $25 \mathrm{~km}^{2}$ \\
\hline Adult male herd range & $81 \mathrm{~km}^{2}$ \\
\hline Movement model & Random, water seeking \\
\hline \multicolumn{2}{|l|}{ For the water seeking model, additional parameters are required } \\
\hline Percentage of cells containing water & $2.5 \%$ \\
\hline Foraging period & $4, \mathbf{6}, 8$ days \\
\hline
\end{tabular}

Herds congregate around permanent water points; therefore concentrations of feral pigs are highest along river systems. We define two movement models for pig herds which may be representative of their behaviour in the wet and dry seasons. In the wet season, when food and water are plentiful, we assume that each herd moves randomly within its home range (the random movement model). In the dry season, each herd has a home range centred on a water source and we model movement such that a herd oscillates between the water location and the edges of its foraging home range (the alternating foraging and water seeking model). The mobility algorithm which implements the water seeking model was inspired by a model for the evacuation of crowds from buildings [31] and is designed such that once a herd has found water, the water source 'repels' the herd for a fixed foraging period after which the herd seeks water again. At each daily time step during the water seeking phase (or every second time step for infectious herds, assuming symptomatic infectivity inhibits mobility by $50 \%$ ), every herd moves to the neighbouring cell which is closest to water or randomly chooses a neighbour if several neighbours are equally close. In contrast, during the foraging phase the herd moves to the neighbour which is furthest from water.

This behaviour is replicated using a potential field method, traditionally employed in robot navigation [3] where the value assigned to each cell by the potential field algorithm is its distance from the nearest water source. To simulate herd movement towards the nearest water location, a herd moves to the neighbouring cell with the lowest value, being the one that is closest to water. Herd mobility alters once it reaches water and then enters the random foraging mode, with herds oscillating between the water source and the edges of their home range.

\subsection{Parameter settings}

In a single simulation, the location and disease state of every herd is traced for the duration of the simulation, as shown in Figure 1. Summary statistics of the number of herds in each disease state are also stored. In Table I we list the set of parameter values used to perform simulations based on pig population data, knowledge of feral pig behaviour and CSF disease parameters [2,5,27,28]. Standard parameter values are indicated by the bold values in Table I. Given the stochastic nature of the disease transmission and movement models, we perform 100 simulations for each simulation experiment.

\section{RESULTS}

The results of 100 simulations using the standard parameter values and random movement model are summarised in Figure 2 where we present the 10th, 50th and 90th percentiles of the daily number of susceptible and infected herds to indicate the variability 


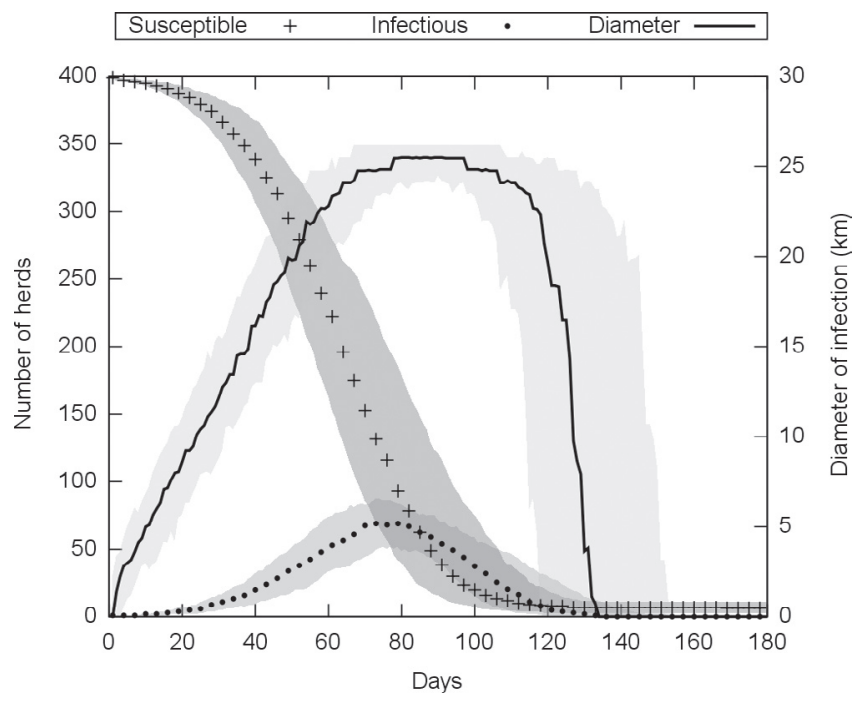

Figure 2. Results of simulations for the random movement model with standard parameter values. The number of herds in the susceptible and infectious states can be read from the left axis. The diameter of the infection can be read from the right axis. The solid line or points is the median of 100 simulations while the upper and lower limits of the shaded region for each state are the 10th and 90th percentiles.

of results. Also shown is the spatial extent of the epidemic, defined as the greatest distance between all pairs of exposed or infectious herds at any given time. Due to uncertainty in knowledge of feral pig population behaviour and CSF transmission properties we performed sensitivity analyses to show the range of potential epidemic behaviours. These sensitivity analyses are shown in Figure 3 where a single parameter is systematically varied using the values in Table I while all other parameters are held fixed to the standard value.

Figure $1 \mathrm{a}$ and $1 \mathrm{~b}$ each illustrate the spatial distribution of pig herds resulting from a single, typical simulation run presented at 10day intervals, where the location of water affects the movement of herds when using the water seeking model. Figure 1a corresponds to a model with only family herds present while Figure 1b corresponds to a mix of family and adult male herds. After an artificial initialisation phase which allows randomly located family herds to cluster around water locations (between day 0 and day 10), they remain close to these locations, only mixing with other herds which co-locate at a water source. By comparison, for a model with $30 \%$ adult male herds (Figure 1b) the epidemic spreads to several water sources.

In Table II we present the median cumulative percentage of infected herds for a selection of different input parameters (see Table I). These numbers quantify the magnitude and duration of the epidemic. The median spatial extent of the epidemic for the same set of models is tabulated in Table III. With the standard parameter values and random movement model, nearly all herds become infected. Using the water seeking model, approximately a third of the herds become infected. Very few herds are infected when the epidemic begins in the wet season (random movement model, herd density $=0.25$ ) when compared with the dry season (water seeking movement model, herd density $=1.0$ ). Inclusion of a relatively small percentage of adult male herds $(15 \%)$ increases the scale, duration (Table II) and spatial extent (Table III) of the epidemic for the water seeking model but not for the random movement model. The length of the foraging phase (four, six or eight days) 

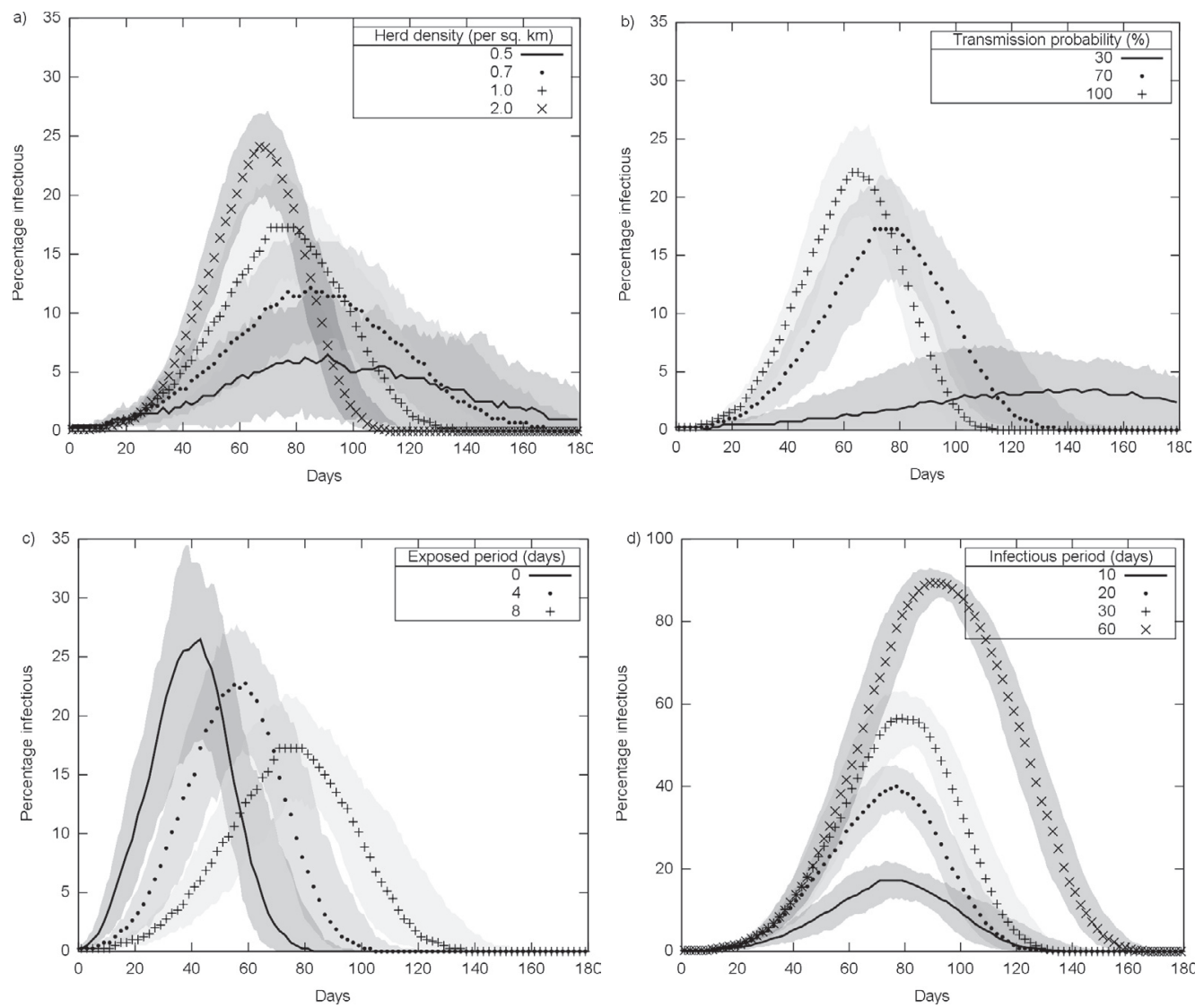

Figure 3. The effect of varying (a) herd density; (b) transmission probability; (c) exposed period; (d) infectious period on the percentage of infectious herds throughout an epidemic. The solid line or points is the median of 100 simulations while the upper and lower limits of the shaded region for each model are the 10th and 90th percentiles. Note that the vertical axis has a different scale for Figure d.

is seen to have little bearing on the magnitude, duration or spatial extent of the epidemic.

In addition to the overall smaller scale of epidemic in the water seeking model, the initial disease spread (i.e. for the first 20 days) is slightly faster with the water seeking model (median cumulative infected percentage of $4.8 \%$ on day 20 ) than the random movement model $(3.5 \%)$ since the locations containing water concentrate herds around them, so that locally the population density is high. In the water seeking model (Table II), this initial spread rate is decreased with increasing percentage of adult male herds $(4.0 \%$ for the model with $30 \%$ adult male herds) which can roam further from the locations containing water, thus reducing the population density at these main contact locations.

\section{DISCUSSION}

Use of the random movement model is seen in Figure 2 to reproduce well known, classical epidemic curves [1] and demonstrates, as expected, their sensitivity to herd density (Figure 3a), to incubation (Figure 3c) and infectious (Figure 3d) periods and to transmission probability (Figure $3 \mathrm{~b}$ ). Random movement generally leads to large epidemics in terms of the number of herds 
Table II. The median cumulative percentage of infected herds for a range of models at 20 day intervals throughout the epidemic. For each model, the median value is obtained from 100 simulations. Standard parameter values (Tab. I) are used with the results for the random and water seeking movement models separated and the herd density indicated in the second column. Variations on the standard parameters are indicated in the first column. The wet season model uses random movement and a herd density of 0.25 . The dry season model uses water seeking movement and a herd density of 1.0.

\begin{tabular}{lccrrrrrrr}
\hline Model and variations & Herd density & \multicolumn{7}{c}{ Cumulative median \% infected herds on epidemic day } \\
\hline Random movement / wet season & & 20 & 40 & 60 & 80 & 100 & \multicolumn{1}{c}{120} & \multicolumn{1}{c}{140} \\
\hline Low density, 0\% adult male & 0.25 & 3.0 & 5.0 & 6.0 & 6.0 & 6.0 & 6.0 & 6.0 \\
0\% adult male & 1.0 & 3.5 & 16.9 & 47.0 & 80.2 & 95.8 & 98.2 & 98.5 \\
30\% adult male & 1.0 & 3.5 & 18.1 & 50.5 & 85.4 & 96.8 & 98.0 & 98.2 \\
& & & & & & & & \\
Water seeking movement / dry season & & & & & & & & \\
\hline 0\% adult male & 1.0 & 4.8 & 16.5 & 24.4 & 27.5 & 30.2 & 30.2 & 31.0 \\
15\% adult male & 1.0 & 4.5 & 16.8 & 28.5 & 37.0 & 41.1 & 44.1 & 47.0 \\
30\% adult male & 1.0 & 4.0 & 17.4 & 32.5 & 43.4 & 51.5 & 55.6 & 57.5 \\
Foraging 4 days & 1.0 & 5.0 & 18.2 & 27.8 & 31.4 & 32.9 & 33.6 & 33.6 \\
Foraging 8 days & 1.0 & 4.2 & 16.4 & 27.1 & 31.5 & 33.4 & 33.4 & 33.4 \\
\hline
\end{tabular}

infected and the area covered except during periods of low population density, which occur at the start of the wet season (Tabs. II and III). In contrast, in the dry period where movement is influenced by the location of water, epidemics are smaller, are concentrated around water locations and show a long distance jump pattern if adult male herds are present.
The demographics of feral pig herds differ between the wet and dry season in two important ways: the herd density is higher at the beginning of the dry season and herds are concentrated around water locations in the dry season but are more evenly distributed in the wet season. The simulation results presented in Table II highlight the difference in scale of an outbreak when comparing early

Table III. The median spatial extent $(\mathrm{km})$ of the epidemic for a range of models at 20 day intervals. Spatial extent is defined as the maximum distance between infected herds. For each model, the median value is obtained from 100 simulations. Standard parameter values (Tab. I) are used with the results for the random and water seeking movement models separated and the herd density indicated in the second column. Variations on the standard parameters are indicated in the first column. The wet season model uses random movement and a herd density of 0.25 . The dry season model uses water seeking movement and a herd density of 1.0 .

\begin{tabular}{|c|c|c|c|c|c|c|c|c|}
\hline Model and variations & Herd density & \multicolumn{7}{|c|}{ Median spatial extent $(\mathrm{km})$ on epidemic day } \\
\hline Random movement / wet season & & 20 & 40 & 60 & 80 & 100 & 120 & 140 \\
\hline Low density, $0 \%$ adult male & 0.25 & 0.5 & 0 & 0 & 0 & 0 & 0 & 0 \\
\hline $0 \%$ adult male & 1.0 & 9.1 & 17.0 & 23.3 & 25.5 & 24.8 & 21.9 & 0 \\
\hline $30 \%$ adult male & 1.0 & 9.1 & 17.8 & 23.7 & 24.8 & 24.1 & 9.9 & 0 \\
\hline
\end{tabular}

Water seeking movement / dry season

\begin{tabular}{lrrrrrrrr}
$0 \%$ adult male & 1.0 & 8.2 & 11.3 & 12.0 & 7.8 & 0 & 0 & 0 \\
$15 \%$ adult male & 1.0 & 8.5 & 12.8 & 16.3 & 15.1 & 9.8 & 0 & 0 \\
$30 \%$ adult male & 1.0 & 10.0 & 14.9 & 18.4 & 19.9 & 18.1 & 13.0 & 0 \\
Foraging 4 days & 1.0 & 9.0 & 11.7 & 12.7 & 7.8 & 0 & 0 & 0 \\
Foraging 8 days & 1.0 & 7.9 & 11.3 & 12.8 & 9.2 & 0 & 0 & 0 \\
\hline
\end{tabular}

Page 8 of 11 (page number not for citation purpose) 
wet (random movement, herd density $=0.25$ ) and early dry season initiation. These results indicate that higher population density makes the start of the dry season the optimal time for a large-scale outbreak of CSF. From this we may determine that eradication will be most difficult if an outbreak occurs at the start of the dry season.

Social groupings of feral pigs influence the distance which herds are able to move, with herds consisting solely of adult males being able to cover greater distances than family herds $[5,16]$. In the dry season, adult male herds, with their larger home ranges, may act as conduits through which the disease is transmitted between otherwise isolated subpopulations by directly connecting them together (see Figure 1). This phenomenon highlights the importance which long-range mobility has on the spatial extent and overall scale of an outbreak by connecting together 'stepping-stone islands' [30]. This connective feature is commonly found in social networks and network analysis techniques developed for human diseases $[6,8,25]$ may also have application here. The results of Table II and Table III show that long-range contact does occur, with adult male herds increasing the area and number of herds infected. The results indicate that almost twice the number of herds become infected when $30 \%$ of the herds are adult male herds, compared with when no adult male herds are present (see Table II). Furthermore, the results indicate that the initial period of the epidemic is relatively insensitive to the presence of adult male herds. This is due to the disease spreading through herds which have common or closely located water sources with the longer-range spread occurring at a later period of the epidemic. The presence of adult male herds also extends the duration of the epidemic as their larger home range results in occasional contact between herds located at different water sources, which then perpetuates the epidemic in a new location. We note that for the random movement model, the percentage of adult male herds has no detectable effect on the time-course of an outbreak (see Table II) and therefore the presence of adult male herds is only significant when their presence increases the inter-herd connectivity of the overall population, as occurs in the dry season. When this occurs, our results suggest that an optimal strategy for disease eradication in the dry season would be to develop culling strategies which initially target adult male herds, so breaking the long-range connections in the contact network.

The lack of accurate data on feral pig demographics and mobility means that some of our assumed parameter values may not be accurate, highlighting the need for obtaining high-quality field data. We have managed this uncertainty by ascertaining parameter sensitivity via simulation experiments which differ by one parameter value, capturing the effect which that parameter has on a complete outbreak, with results presented in Tables II and III, and Figure 3.

This study presents techniques developed for modelling diseases where the host population is highly mobile, as with a wild or feral species. In this study we have two different herd types (family and adult male) of the same species, but the method may also be applied to situations where different species with different mobility characteristics may mix and be affected by the same disease, such as with foot-and-mouth disease. The explicit mobility modelling method may also be applicable for capturing the movement of migratory wildlife and the effect which such seasonal movement has on disease spread to domestic animal populations which share specific diseases and habitat with the migratory species. Related simulation models, also with explicit host mobility, have recently been developed to model the spread of human diseases such as pandemic influenza $[9,10,14,15,23]$ and smallpox [8].

Acknowledgements. We acknowledge funding support from the Australian Research Council, the Department of Agriculture and Food (Western Australia), the Danish Food and Veterinary Research Institute and from a National ICT Australia Fellowship to GJM. The two anonymous reviewers are thanked for their diligent and helpful reviews. 


\section{REFERENCES}

[1] Anderson R.M., May R.M., Infectious diseases of humans: dynamics and control, Oxford University Press, Oxford, UK, 1991.

[2] Artois M., Depner K.R., Guberti V., Hars J., Rossi S., Rutili D., Classical Swine Fever (hog cholera) in wild boar in Europe, Rev. Off. Int. Epizoot. (2002) 21:287-303.

[3] Barraquand J., Langlois B., Latombe J.C., Numerical potential field technique for robot path planning, IEEE Transactions on System Man and Cybernetics (1992) 22:224-241.

[4] Bates T.W., Thurmond M.C., Carpenter T.E., Description of an epidemic simulation model for use in evaluating strategies to control an outbreak of foot-and-mouth disease, Am. J. Vet. Res. (2003) 64: 195-204.

[5] Caley P., Movements, activity patterns and habitat use of feral pigs (Sus Scrofa) in a tropical habitat, Wildl. Res. (1997) 24:77-87.

[6] Christley R.M., Pinchbeck G.L., Bowers R.G., Clancy D., French N.P., Bennett R., Turner J., Infection in social networks: using network analysis to identify high risk individuals, Am. J. Epidemiol. (2005) 162:1024-1031.

[7] Doran R.J., Laffan S.W., Simulating the spatial dynamics of foot and mouth disease outbreaks in feral pigs and livestock in Queensland, Australia, using a susceptible-infected-recovered cellular automata model, Prev. Vet. Med. (2005) 70:133-152.

[8] Eubank S., Guclu H., Anil Kumar V.S., Marathe M.V., Srinivasan A., Toroczkai Z., Wang N., Modelling disease outbreaks in realistic urban social networks, Nature (2004) 429:180-184.

[9] Ferguson N.M., Cummings D.A., Fraser C., Cajka J.C., Cooley P.C., Burke D.S., Strategies for mitigating an influenza pandemic, Nature (2006) 442:448-452.

[10] Ferguson N.M., Donnelly C.A., Anderson R.M., The foot-and-mouth epidemic of Great Britain: pattern of spread and impact of interventions, Science (2001) 292:1155-1160.

[11] Ferguson N.M., Donnelly C.A., Anderson R.M., Transmission intensity and impact of control policies on the foot and mouth epidemic in Great Britain, Nature (2001) 413:542-548.

[12] Fu S., Milne G., A Flexible automata model for disease simulation, in: Lecture notes in computer science 3305, Springer, Berlin, Heidelberg, Germany, 2004, pp. 642-649.

[13] Garner M.G., Whan I.F., Gard G.P., Phillips D., The expected economic impact of selected exotic diseases on the pig industry of Australia, Rev. Off. Int. Epizoot. (2001) 20:671-685.

[14] Germann T.C., Kadau K., Longini I.M. Jr., Macken C.A., Mitigation strategies for pandemic influenza in the United States, Proc. Natl. Acad. Sci. USA (2006) 103:5935-5940.

[15] Glass R.J., Glass L.M., Beyeler W.E., Min H.J., Targeted social distancing design for pandemic influenza, Emerg. Infect. Dis. (2006) 12:1671-1681.

[16] Heise-Pavlov S., Heise-Pavlov P., Bradley A., Carpal glands in feral pigs (Sus domesticus) in tropical lowland rainforest in north-east Queensland, Australia, J. Zool. (2005) 266:73-80.

[17] Highfield L., Ward M.P., Laffan S.W., Representation of animal distributions in space: how geostatistical estimates impact simulation modeling of foot-and-mouth disease spread, Vet. Res. (2008) 39:17.

[18] Hone J., How many feral pigs in Australia, Australian Wildlife Research (1990) 17:571-572.

[19] Jalvingh A.W., Nielen M., Maurice H., Stegeman A.J., Elbers A.R., Dijkhuizen A.A., Spatial and stochastic simulation to evaluate the impact of events and control measures on the 1997-1998 classical swine fever epidemic in The Netherlands, Prev. Vet. Med. (1999) 42:271-295.

[20] Keeling M.J., Rohani P., Estimating spatial coupling in epidemiological systems: a mechanistic approach, Ecol. Lett. (2002) 5:20-29.

[21] Keeling M.J., Woolhouse M.E., May R.M., Davies G., Grenfell B.T., Modelling vaccination strategies against foot-and-mouth disease, Nature (2003) 421:136-142.

[22] Kermack W., McKendrick A., A contribution to the mathematical theory of epidemics, Proc. R. Soc. Lond. A Biol. Sci. (1927) 115:700-721.

[23] Longini I.M.Jr., Nizam A., Xu S., Ungchusak K., Hanshaoworakul W., Cummings D.A.T., Halloran M.E., Containing pandemic influenza at the source, Science (2005) 309:1083-1087.

[24] McVicar J., Sutmoller P., Ferris D.H., Campbell C.H., Foot-and-mouth disease in white-tailed deer: clinical signs and transmission in the laboratory, Proc. Annu. Meet. US Anim. Health Assoc. (1974) 169-180.

[25] Meyers L.A., Newman M.E., Pourbohloul B., Predicting epidemics on directed contact networks, J. Theor. Biol. (2006) 240:400-418.

[26] Morris R.S., Wilesmith J.W., Stern M.W., Sanson R.L., Stevensen M.A., Predictive spatial modelling of alternative control strategies for the foot-and-mouth disease epidemic in Great Britain, 2001, Vet. Rec. (2001) 149:137-144. 
[27] Pech R.P., Hone J., A model of the dynamics and control of foot and mouth disease in feral pigs in Australia, J. Appl. Ecol. (1988) 25:63-77.

[28] Pech R.P., McIlroy J.C., A model of the velocity of advance of foot and mouth disease in feral pigs, J. Appl. Ecol. (1990) 27:635-650.

[29] Pinto A.A., Foot-and-mouth disease in tropical wildlife, Ann. NY Acad. Sci. (2004) 1026:65-72.

[30] Renshaw E., Modelling Biological Populations in Space and Time, Cambridge University Press, Cambridge, UK, 1993.
[31] Thompson P.A., Marchant E.W., A computer model for the evacuation of large buildilng populations, Fire and Safety Journal (1995) 24:131148.

[32] Tildesley M.J., Savill N.J., Shaw D.J., Deardon R., Brooks S.P., Woolhouse M.E., et al., Optimal reactive vaccination strategies for a foot-and-mouth outbreak in the UK, Nature (2006) 440:83-86.

[33] Ward M.P., Laffan S.W., Highfield L.D., The potential role of wild and feral animals as reservoirs of foot-and-mouth disease, Prev. Vet. Med. (2007) 80:9-23. 